$\mathrm{AB} 1001$ CHANGES IN THE PARATHYROID HORMONE LEVEL IN CHILDREN WITH JUVENILE IDIOPATHIC ARTHRITIS

N. Shevchenko ${ }^{1,2}$, Y. Khadzhynova ${ }^{1,2} .{ }^{1}$ V.N. Karazin Kharkiv National University, Department of Pediatrics No 2, Kharkiv, Ukraine; ${ }^{2}$ SI Institute for Children and Adolescents Health Care of NAMS of Ukraine, Department of Cardiorheumatology, Kharkiv, Ukraine

Background: It is proved that rheumatic diseases are accompanied by pronounced changes in calcium-phosphorus metabolism, which underlies the development of osteopenia syndrome. If in previous years glucocorticosteroid therapy (GCS) was considered to be the main reason for this, then the role of pro-inflammatory agents (activity of the pathological process), provision with vitamin $D(V i t D)$, and the effect of basic therapy are currently being discussed. It is also known that a decrease blood level of vitamin $\mathrm{D}$ leads to a violation of the absorption of calcium and phosphorus, a further increase in the level of parathyroid hormone, which underlies the risk of a decrease in bone mineral density. Objectives: To study the level of parathyroid hormone in children with juvenile idiopathic arthritis, its relationship with the course of the disease and vitamin D status.

Methods: 91 patients with JIA (61 girls and 30 boys), with polyarticular ( $\mathrm{n}=$ $41)$, oligoarticular $(n=29)$ and undifferentiated $(n=18)$ variants of JIA were examined. The age of the patients was $10.5 \pm 1.7$ years. The duration of the disease was $4.1 \pm 1.1$ years. All children receive basic methotrexate therapy. The control group included 25 peers of the corresponding gender. JADAS27 was counted, the levels of 25-hydroxycalciferol (25-OH D) and PTH were determined by chemiluminescent method. Corresponded to the content of vitamin $\mathrm{D}$ in blood serum, the normal level was noted in 14 patients, insufficiency - in 41 patients, deficiency - in 32 patients.

Results: The level of PTH in children with JIA remained within physiological values $(30.6 \pm 2.1 \mathrm{pg} / \mathrm{ml}$; from 12.7 to $61.8 \mathrm{pg} / \mathrm{ml})$ despite the high frequency of a VitD decrease in blood (80.2\%). The level of PTH was not significant in groups of patients with a different level of vitamin $\mathrm{D}(32.8 \pm 2.1 \mathrm{pg} / \mathrm{ml}$ at deficiency group; $29.2 \pm 2.4 \mathrm{pg} / \mathrm{ml}$ at insufficiency group; $29.1 \pm 1.8 \mathrm{pg} / \mathrm{ml}$ at a normal level of VitD group). PTH was comparable and did not differ in children of different sexes. The highest level of PTH was in patients with a polyarticular variant of JIA (34.2 $\pm 4.5 \mathrm{pg} / \mathrm{ml}, \mathrm{p}<0.05)$, which was accompanied by the lowest level of vitamin $\mathrm{D}(20.9 \pm 2.1 \mathrm{ng} / \mathrm{ml}, \mathrm{p}<0,05)$ in the same group. There was obtained reliable correlations of activity indicators (JADAS27) taking into account Vit D and PTH, as well as with diseases duration and age of patients $(p<0.05)$.

Conclusion: A study of the level of PTH in children with JIA did not show a significant increase depending on the vitamin $D$ status in these patients. However, the age-related state of PTH is associated with the activity of the pathological process, the prevalence of articular syndrome and prolonged illness.

Disclosure of Interests: None declared

DOI: 10.1136/annrheumdis-2020-eular.6499

\section{AB1002 IS KAWASAKI DISEASE OR SYSTEMIC JUVENILE IDIOPATHIC ARTHRITIS IN CHILDREN WITH FEVER WITHOUT A SOURCE?}

B. Sözeri ${ }^{1}$, F. Demir ${ }^{2}$, T. Merter ${ }^{3}$, M. Karacan ${ }^{4} .{ }^{1}$ istanbul Health Sciences University, Pediatric rheumatology, Istanbul, Turkey; ${ }^{2}$ Istanbul Health Sciences University, Pediatric rheumatology, Istanbul, Turkey; ${ }^{3}$ Istanbul Health Sciences University, Pediatrics, Istanbul, Turkey; ${ }^{4}$ Istanbul Health Sciences University, Pediatric Cardiology, Istanbul, Turkey

Background: Fever without a source (FWS) is caused by various diseases, making differential diagnosis difficult. Clinical similarities between Kawasaki disease (KD) and systemic Juvenile Idiopathic Arthritis (sJIA) are well known. Kawasaki disease (KD), a self-limiting systemic vasculitis, remains of unknown etiology and can cause irreversible coronary artery aneurysms (CAAs). SoJIA is sometimes confused with incomplete KD because both diseases have overlapping clinical features and can be accompanied with CAAs and/or SJIA with macrophage activation syndrome (MAS).

Objectives: In this study, the frequency of both KD and SJIA among the patients evaluated with FWS and the clinical features of patients diagnosed with Kawasaki disease.

Methods: Medical records of patients who first visited our department between January 2016 and December 2019 were reviewed

Results: A total of 107 patients were enrolled in this study, including 43 patients $(40.2 \%, 23$ males) who fulfilled the criteria of Kawasaki disease and 64 patients $(59.8 \%, 39$ males) who did not fulfill them. In patients who fulfilled the criteria of classical FWS, 36(33.6\%, 20 males) patients were diagnosed with systemic juvenile idiopathic arthritis. The mean age of the patients with Kawasaki disease was $30.0 \pm 20,4$ months (median 25 months), the mean age of other patients was $52,6 \pm 40$ months (median 39,5 months). The mean age of the patients with sJIA patients was $87,6 \pm 49,8$ (median 80 months). Kawasaki patients were younger than others $(p=0.01)$. There was no difference in gender between groups.

In Kawasaki patients, the most common clinical feature at diagnosis was fever $(100 \%)$ followed by conjunctival congestion and mucosal changes $(69 \%)$. The last two findings are more significant in kawasaki patients than others $(p<0,00)$. Twenty-six (59\%) patients had completed KD while $25 \%$ had incomplete KD. $7(16 \%)$ patients had atypical KD. The mean fever duration was longer in SJIA patients than KD and others (median 14,8 and 7 days, $p<0.00$ ). All patients with $\mathrm{KD}$ received IVIG $(2 \mathrm{~g} / \mathrm{kg}$, infusion in $12 \mathrm{~h})$ and aspirin $(60 \mathrm{mg} / \mathrm{kg} / \mathrm{day}) .13 .6 \%$ of the patients also received oral corticosteroids because of IVIG resistance. Thirty-one patients $(72.1 \%)$ responded to IVIG treatment, whereas 12 (6 female, 6 male) were IVIG resistant. CAI was detected in echocardiography at diagnosis in $10(22.7 \%)$ (6 female; 4 male) patients. We also detected 4 patients pericarditis with /without CIA

Conclusion: The clinical presentations of KD and SJIA are quite similar with fever, rash, hepatomegaly, and lymphadenopathy. All 2 entities may provide clues to potentially shared immunopathology.

References:

[1] Arslanoglu Aydin E et al. The factors affecting the disease course in Kawasaki disease. Rheumatol Int. 2019 Aug;39(8):1343-1349

[2] Dong $S$ et al. Diagnosis of systemic-onset juvenile idiopathic arthritis after treatment for presumed Kawasaki disease. J Pediatr. 2015 May;166(5): 1283-8.

Disclosure of Interests: None declared

DOI: 10.1136/annrheumdis-2020-eular.4689

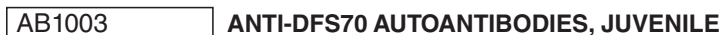 IDIOPATHIC ARTHRITIS (JIA) AND UVEITIS: ARE ANTI- DFS70 A PREDICTIVE MARKER OF UVEITIS RISK IN JIA PATIENTS?}

E. Tadiotto ${ }^{1}$, C. Mansoldo ${ }^{2}$, G. Bellisola ${ }^{1}$, F. Caldonazzi ${ }^{3}$, G. Aiello ${ }^{1}$, G. Melotti ${ }^{1}$, M. Maschio ${ }^{1}$, E. Giacomelli ${ }^{1}$, S. Pieropan ${ }^{1}$. ${ }^{1}$ Azienda Ospedaliera Universitaria Integrata Verona, Pediatrics, Verona, Italy; ${ }^{2}$ Azienda Ospedaliera Universitaria Integrata Verona, Ophthalmology, Verona, Italy; ${ }^{3}$ Ospedale di Rovereto,

Pediatrics, Rovereto, Italy

Background: Juvenile idiopathic arthritis (JIA) is the most common paediatric rheumatic disease. Its most threatening complication is represented by uveitis, which could cause severe visual impairment if not diagnosed and treated promptly. It is an asymptomatic uveitis and the diagnosis is only instrumental. Therefore, regular ophthalmologic surveillance is crucial in the management of JIA. To date there are no specific predictive markers of uveitis development among JIA patients, including serologic subsets. Anti-Nuclear Antibodies (ANA) positive patients have the highest risk of iridocyclitis, but ANA are not specific They could be found in patients with JIA without uveitis, in many other rheumatologic and inflammatory conditions and also in healthy subjects $(6-12 \%$ of children). Anti-DFS70 antibodies (ANA forming a specific pattern in immunofluorescence) have been taken into consideration, but their role has not yet been established in a pediatric setting. Currently, there are few reports, involving small groups of patients and with non-univocal results. Some studies report antiDFS70 antibodies more frequently in children with rheumatological diseases, in particular JIA-related uveitis; on the contrary, others describe them in healthy ANA positive patients.

\section{Objectives:}

1) To evaluate the correlation between anti-DFS70 autoantibodies and the risk of developing uveitis in a cohort of patients with JIA ANA + in pediatric age;

2) to compare the prevalence of anti-DFS70 in patients with JIA, with a group of healthy ANA + children, to better define the role of these autoantibodies (potential risk factor or a protective factor for the development of AARDs in children?)

Methods: We evaluated retrospectively 51 patients with JIA ANA +. We divided these patients in two groups, according to the presence $(n=11)$ or the absence $(n=40)$ of uveitis. For each patient we evaluated: gender, current age, age at diagnosis, type of JIA, therapy, presence of other diseases, dosage of ANA (with IF-Hep2), research of anti-ENA and anti-DFS70 antibodies (with chemiluminescence). Subsequently the whole group of patients with JIA was compared with a control group of healthy subjects aged $\leq 18$ years $(n=30)$, followed in the pediatric rheumatology clinic for occasional finding of ANA positivity, without pathologies at the moment of the study (in particular without rheumatological or autoimmune diseases).

Results: Among patients with JIA without uveitis, anti-DFS70 autoantibodies were positive only in one patient. Anti-DFS70 were negative in all patients with JIA and uveitis. The difference between the two groups is not significant $(p=1)$. In the group of healthy patients $6 / 30(20 \%)$ presented a positivity of anti-DFS70 autoantibodies, in the absence of anti-ENA.

Conclusion: Our data revealed no correlation between the positivity of antiDFS70 autoantibodies and the risk of uveitis in patients with JIA ANA +, even 
if the number of patients is small. Further studies are needed to identify a reliable predictive marker of uveitis risk in JIA patients. The finding of a significant greater prevalence of anti-DFS70 autoantibodies in healthy ANA + subjects allows to suppose that this autoantibody could represent a possible protective marker for development of AARDs in asymptomatic children with isolated ANA positivity, as for adults. To confirm this hypothesis, it would be useful to carry on the study prospectively, encompassing children with other rheumatological diseases, and prolonging the clinical and laboratory follow up.

References:

[1] Clarke S, Sen ES, Ramanan AV. Juvenile idiopathic arthritis-associated uveitis. Ped Reumatol 2016; 14: 27.

[2] Seeling CA, Bauer O, Seeling H-P. Autoantibodies Against DFS70/LEDGF Exclusion Markers for Systemic Autoimmune Rheumatic Diseases (SARD). Clin. Lab 2016;62:499- 517.

[3] Schmeling $\mathrm{H}$ et al. Autoantibodies to Dense Fine Speckles in Pediatric Diseases and Controls. The Journal of Rheumatology 2015;42(12):2419-2426.

Disclosure of Interests: None declared

DOI: 10.1136/annrheumdis-2020-eular.1815

\section{AB1004 JUVENILE DERMATOMYOSITIS (JDM) IN SOUTHEAST ASIA: A 20-YEAR SINGAPORE EXPERIENCE}

K. L. Teh ${ }^{1}$, L. Das ${ }^{1}$, Y. X. Book ${ }^{1}$, T. Arkachaisri ${ }^{1,2} .{ }^{1} K K$ Women's and Children's Hospital, Rheumatology and Immunology, Singapore, Singapore, ' ${ }^{2}$ Duke-NUS Medical School, Singapore, Singapore

Background: Juvenile dermatomyositis (JDM) is a multisystem inflammatory disease of childhood with variable demographics, clinical features and outcomes. No studies have described the characteristics of JDM patients from Southeast Asia population.

Objectives: To describe the clinical characteristics and outcomes of JDM patients in Singapore over a 20 -year period.

Methods: Patients diagnosed with JDM from 1999 to 2019 at KK Women's and Children's Hospital, Singapore, were recruited. Nonparametric descriptive statistics were used to described data. Kaplan-Meier analyses were used to estimate the probability of remission. Multivariate logistic and Cox regression analyses were used to determine predictors as appropriate. The significant level was set at $<0.05$

Results: 32 JDM were identified. Clinical characteristics and treatment used are shown in Table 1.

\begin{tabular}{lccc}
\hline & All $(\mathrm{n}=32)$ & Monophasic $(\mathrm{n}=17)$ & Polyphasic $(\mathrm{n}=14)$ \\
\hline Male & $14(43.8)$ & $7(41.2)$ & $7(50.0)$ \\
Age at onset (yrs)* & $6.4(4.5-9.8)$ & $5.4(4.1-8.5)$ & $7.4(5.6-12.3)$ \\
Lag period (mo) & $3.5(1.0-12.5)$ & $2.0(1.0-16.8)$ & $6.8(1.2-15.8)$ \\
Heliotrope & $16(50)$ & $9(52.9)$ & $7(50)$ \\
Gottron papule & $23(71.9)$ & $12(70.6)$ & $11(78.6)$ \\
Malar rash & $19(61.3)$ & $9(52.9)$ & $10(71.4)$ \\
Vasculitic rash & $19(61.3)$ & $8(47.1)$ & $11(78.6)$ \\
Arthritis & $10(31.3)$ & $3(17.6)$ & $7(50)$ \\
Nailfold changes & $28(87.5)$ & $15(88.2)$ & $13(92.9)$ \\
Calcinosis & $9(28.1)$ & $4(23.5)$ & $5(35.7)$ \\
Positive ANA & $17(53.1)$ & $10(58.8)$ & $7(50)$ \\
Positive Myositis antibodies & $4(12.5)$ & $2(11.8)$ & $2(14.3)$ \\
Laboratory at diagnosis, U/L ${ }^{*}$ & & & \\
CK & $324(134-2229)$ & $746(139-2965)$ & $351(155-2622)$ \\
LDH & $650(450-943)$ & $650(398-1015)$ & $714(512-944)$ \\
ALT & $28(16-106)$ & $35(18-109)$ & $38(15-104)$ \\
AST & $50(30-108)$ & $50(31-113)$ & $85(36-215)$ \\
Aldolase & $13.7(8.1-28.0)$ & $14.2(8.4-26.6)$ & $16.3(8.6-38.5)$ \\
CMAS score at diagnosis & $29(21-43)$ & $28(21-35)$ & $35(11-43)$ \\
\hline
\end{tabular}

${ }^{*}$ median (IQR), otherwise - n (\%)

Pulse methylprednisolone (pMP) was used in $53.1 \%$ of patients after diagnosis. Median time to inactive disease (ID) was 5.3 months (IQR $2.8-12.8$ ). Male, older age and patients on pMP ( $p=0.003-0.044)$ achieved ID sooner. Older patients also developed disease flare sooner after achieving ID ( $p$ $=0.024)$. No clinical features nor lab investigations predicted JDM disease course. Malay patients was associated with higher risk of calcinosis $(p=$ 0.017).

Compared to adult dermatomyositis patients in Singapore ${ }^{1}$, our cohort had more cutaneous manifestations including malar rash, vasculitic rash and nailfold changes. Table 2 shows the time for each muscle enzymes to normalise.

\begin{tabular}{lc}
\hline CK & $1.49(0.69-3.53)$ \\
LDH & $4.53(2.35-25.04)$ \\
ALT & $1.71(0.90-4.13)$ \\
AST & $0.97(0.53-3.12)$ \\
Aldolase & $3.12(2.35-8.30)$
\end{tabular}

median in months (IQR)

Conclusion: Our cohort of JDM patients had more calcinosis compared to othe Asian population ${ }^{2}$. Malay population is at higher risk of this complication. It is crucial to achieve ID state in the shortest time possible to avoid significant morbidity. Our study suggests that early treatment with pMP is associated with shorter time to ID. There is no predictor identified for disease course, similar to previous studies $^{3}$.

\section{References:}

[1] Liu, Wen Chun, et al. "An 11-year review of dermatomyositis in Asian patients." Annals Academy of Medicine Singapore 39.11 (2010): 843.

[2] Sun, Chi, et al. "Juvenile dermatomyositis: a 20-year retrospective analysis of treatment and clinical outcomes." Pediatrics \& Neonatology 56.1 (2015) 31-39.

[3] Stringer, Elizabeth, Davinder Singh Grewal, and Brian M. Feldman. "Predicting the course of juvenile dermatomyositis: significance of early clinical and laboratory features." Arthritis \& Rheumatism: Official Journal of the American College of Rheumatology 58.11 (2008): 3585-3592.

Disclosure of Interests: None declared

DOI: 10.1136/annrheumdis-2020-eular.5929

\section{AB1005 BULLOUS LUPUS (BSLE) AS THE FIRST MANIFESTATION OF SYSTEMIC LUPUS ERYTHEMATOSUS IN THE PEDIATRIC POPULATION (PSLE): A DIAGNOSTIC CHALLENGE IN DAILY PRACTICE}

F. Torres ${ }^{1}$, M. Velasquez ${ }^{1,2}$, L. Ramirez ${ }^{3}$, L. Leon ${ }^{4}$, M. Martinez ${ }^{1} .{ }^{1}$ Universidad de Antioquia, Medellin, Colombia; ${ }^{2}$ Hospital San Vicente Fundacion, Medellin Colombia; ${ }^{3}$ Hospital Fundacion Valle del Lili, Cali, Colombia; ${ }^{4}$ Universidad Nacional de Colombia, Bogota, Colombia

Background: Cutaneous manifestations are observed in $59-85 \%$ of patients with SLE but less than $5 \%$ developed BSLE. In the GLADEL cohort, the prevalence is $0.41 \%$. BSLE literature in children is scarce
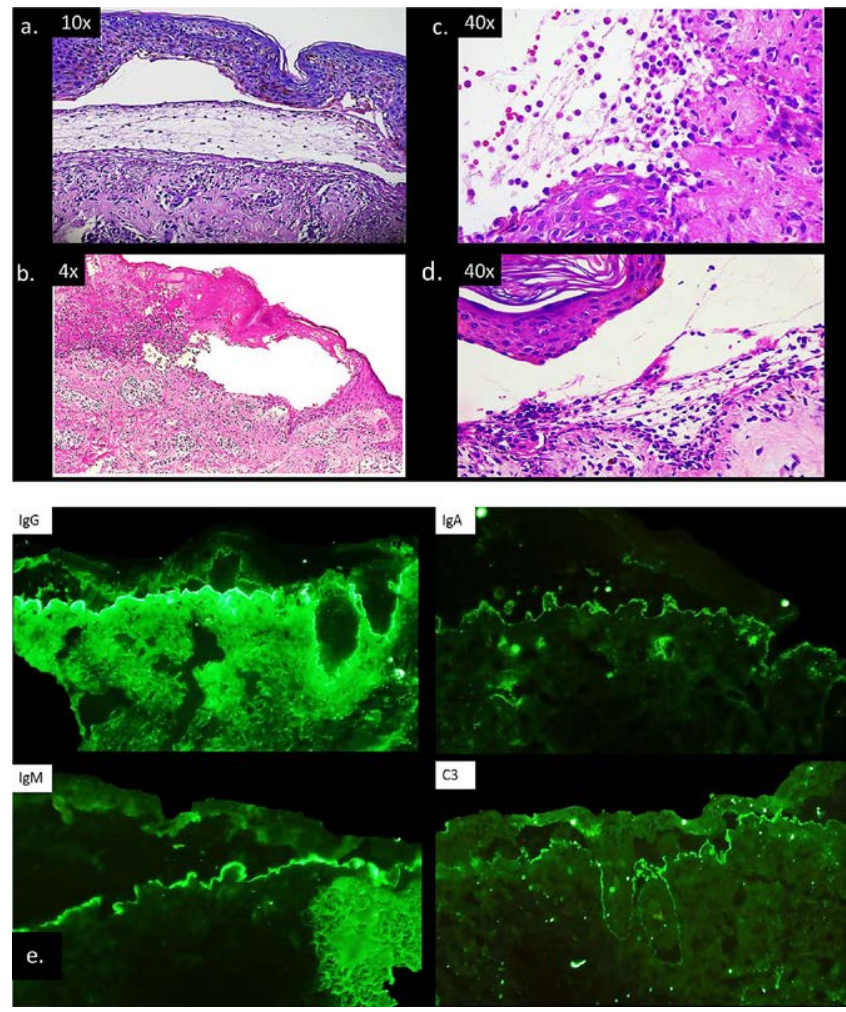

Fig 1. Histology and DIF of BSLE H-E $\mathbf{a}$ and $\mathbf{b}$ : subepidermal blisters $\mathbf{c}$ and $\mathbf{d}$ : abundant neutrophils are observed e: DIF full house linear pattern 